\title{
Reliable Global Navigation System using Flower Constellation
}

\author{
Daniele Mortari*, Jeremy J. Davis ${ }^{\dagger}$, Ashraf Owis ${ }^{\ddagger}$ and Hany Dwidar ${ }^{\S}$ \\ *Professor, 746C H.R. Bright Bldg, Aerospace Engineering, \\ Texas A\&M University, College Station, \\ TX 77843-3141, Tel.: (979) 845-0734, Fax: (979) 845-6051, \\ AIAA Associate Fellow. \\ E-mail: mortari@tamu.edu \\ †VectorNav Technologies, \\ LLC, Richardson, TX 75081, \\ E-mail jeremy.davis@tamu.edu \\ $\ddagger$, Cairo, 13126 (Egypt) \\ E-mail: aowis@eun.eg \\ $\S$, Cairo, 13126 (Egypt) \\ E-mail: hrydwidar@gmail.com
}

\begin{abstract}
For many space missions using satellite constellations, symmetry of satellites distribution plays usually a key role. Symmetry may be considered in space and/or in time distribution. Examples of required symmetry in space distribution are in Earth observation missions (either, for local or global) as well as in navigation systems. It is intuitive that to optimally observe the Earth a satellite constellation should be synchronized with the Earth rotation rate. If a satellite constellation must be designed to constitute a communication network between Earth and Jupiter, then the orbital period of the constellation satellites should be synchronized with both Earth and Jupiter periods of revolution around the Sun. Another example is to design satellite constellations to optimally observe specific Earth sites or regions. Again, this satellites constellation should be synchronized with Earth's rotational period and (since the time gap between two subsequent observations of the site should be constant) also implies time symmetry in satellites distribution. Obtaining this result will allow to design operational constellations for observing targets (sites, borders, regions) with persistence or assigned revisit times, while minimizing the number of satellites required.
\end{abstract}

Constellations of satellites for continuous global or zonal Earth coverage have been well studied over the last twenty years, are well known and have been well documented [1], [2], [7], [8], [11], [13]. A symmetrical, inclined constellation, such as a Walker constellation [1], [2] provides excellent global coverage for remote sensing missions; however, applications where target revisit time or persistent observation are important lead to required variations of traditional designs [7], [8]. Also, few results are available that affect other figures of merit, such as continuous regional coverage and the systematic use of eccentric orbit constellations to optimize"hang time" over regions of interest. Optimization of such constellations is a complex problem and the general-purpose constellation design methodology used today is largely limited to Walker-like constellations.

As opposed to Walker Constellations [1], [2], which were looking for symmetries in inertial reference frame, Flower Constellations [11] were devised to obtain symmetric distributions of satellites on rotating reference frames (e.g., Earth, Jupiter, satellite orbit). Since the theory of Flower Constellations has evolved with time the next section is dedicated to the summary of the theory up to the current status. The FCs solution space has been recently expanded with the Lattice theory [13], [14], encompassing all possible symmetric solutions.

\section{Flower Constellations Theory}

The FC theory, devised and developed at Texas A\&M [11], is a natural extension of the theory of compatible orbits. When an orbit is compatible, the satellite trajectory in the rotating frame becomes a closed-loop trajectory. The original theory defines a Flower Constellation a set of $N_{s}$ satellites following the same (closed) trajectory with respect to a rotating reference frame fixed to the Earth. This condition implies

1) The period of revolution, $T_{p}$, of each satellite about the Earth is a rational multiple of the period of rotation of the Earth, $T_{d}$. That is, $N_{p} T_{p}=N_{d} T_{d}$ for some positive (coprime) integers $N_{d}$ and $N_{p}$.

2) The orbital parameters $a, e, i$ and $\omega$ are the same for all the satellites.

3) The mean anomaly at epoch $M_{i}$ and the right ascension of the ascending node $\Omega_{i}$ of the orbit of each satellite satisfy $N_{p} \Omega_{i}=-N_{d} M_{i} \bmod (2 \pi)$.

The first item guarantees that the trajectory in the rotating frame is closed (loop completed by the repetition period, $\left.T_{\text {rep }}=N_{p} T_{o}=N_{d} T_{\oplus}\right)$. In particular, in the Earth-Centered Earth-Fixed (ECEF) rotating frame, the compatible orbit becomes a repeating ground-track orbit.. The second and third item are necessary and sufficient conditions to have all the satellites on the same trajectory (a complete proof of this fact is given in [32]). To capture the key idea of FCs let's consider Fig. 1. This figure shows a FC made of 4 satellites moving in 8 hr equatorial orbits with the four major axes orthogonal. Since $24 / 8=3$, each satellite passes through 3 apogees per day. By judicious phasing, the satellites all move on the same EarthFixed (EFEC) relative trajectory (red curve with the 3 apogees $120^{\circ}$ apart in Fig. 1). Furthermore, the three apogee loops in an ECEF frame are traversed very slowly with a hang time of 


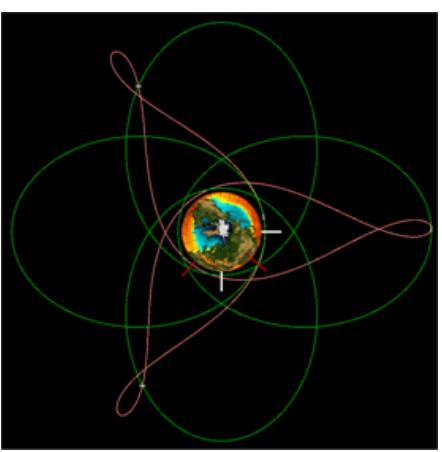

Fig. 1. All 4 satellites are on the same space track (red trajectory; fixed in ECEF).

about $5.3 \mathrm{hr}$ for each satellite to traverse its 3 apogee loops per day, therefore each of the 4 satellites spends about 2/3 of a day near apogee. Notice these loops lie in a small region in ECEF, and through intelligent design, 3 satellites are always in the apogee loops, and the 4-th is en-route from perigee to replace the satellite ready to exit the apogee loop. This pattern of always having 3 satellites near apogee enables 24/7 persistence over three regions with only 4 MEO satellites. This is one of an infinite family of possibilities, and optimizing over these constellations is the first step of the proposed research.

\section{A. From original theory to Lattice theory}

In addition to a resonant period $T_{p}=\frac{N_{d}}{N_{p}} T_{d}$ we can impose the relation $N_{p} \Omega=-N_{d} M$ for all satellites. This condition gives some degrees of freedom for selecting the locations of the satellites in the $(\Omega, M)$-space, to get symmetries in space by using phasing parameters $\left(F_{n}, F_{d}\right.$, and $\left.F_{h}\right)$. This procedure allows a maximum of $N_{d} F_{d} / G$ satellites in a FC, where $G=$ $\operatorname{gcd}\left(N_{d}, N_{p} F_{n}+F_{d} F_{h}\right)$. A FC with the maximum number of satellites allowed by the previous formula is called a Harmonic FC (HFC). The satellites in a HFC exhibit a shape-preserving dynamic, thus behaving like a true rigid body in space. The use of phasing parameters to design FC and HFC is simple, but some number-theoretic problems have been recently found [28] to generalize the theory. The following list describes some of these problems:

1) Equivalency Problem. Many combinations of input parameters can give the same FC. For HFC a complete solution of this problem has been found [1]. It has been shown that 3 invariants are sufficient to uniquely characterize them: the number of satellites per orbit $N_{s o}$, the number of orbits $N_{o}=F_{d}$, and a configuration number, $N_{c}$. Formulas to compute these invariants are known.

2) Similarity Problem. Two equivalent HFC with a different $N_{p} / N_{d}$ ratio may have the same relative dynamics while rotating at different velocities. Reference [1] provides a complex algorithm to compute all similar (homotetic) HFC with given invariants $\left(N_{s o}, N_{o}, N_{c}\right)$.

3) Geometric properties of HFC. The number of perigees and apogees of a HFC is time invariant. How to compute these geometric invariants requires further research. This problem is connected with the problem of computing the number of axial symmetries of rigid bodies.

4) Time and space uniform FC. The satellites of a FC are all located in a single closed-loop trajectory in the rotating frame that depends only on the ratio $N_{d} / N_{p}$. By dividing this relative orbit in equally spaced time intervals, we obtain a FC whose time gap between 2 subsequent observations of the same target is constant (time-uniform). This is achieved by introducing a new mean anomaly (Flower Anomaly) to map the $(\Omega, M)$ space in time [1] by direct application of the Chinese Remainders Theorem. Space-uniform FCs for worldwide targets are obtained through optimization of the Thompson problem (uniformly points distribution over a sphere).

All these problems have been addressed using the phasing parameters has and have been recently solved [9] with a new FC theory (Lattice FC). The new approach decouples the compatibility condition and the shape parameters. The -space satellite locations (mathematically described as a torus) are given by all the solutions of a modular system of equations.

$$
L P_{k}=\left[\begin{array}{ll}
a & b \\
c & d
\end{array}\right]\left\{\begin{array}{l}
\Omega_{k} \\
M_{k}
\end{array}\right\}=\left\{\begin{array}{l}
0 \\
0
\end{array}\right\} \bmod (2 \pi)
$$

The four integer parameters in these equations $(a, b, c, d)$ give a new meaningful matrix associated with the FC. The number of orbits $N_{\Omega}$, the number of satellite per orbits $N_{s o}$, and the configuration number $N_{c}$ can be derived from the Lattice matrix $(L)$ by computing it Smith-Hermite normal form $H=\left[\begin{array}{cc}N_{\Omega} & 0 \\ N_{c} & N_{\text {so }}\end{array}\right]$ where $\quad H=L U, \quad$ and $\quad U \in G L_{2}(\mathbb{Z})$

All the possible ratios $N_{d} / N_{p}$ for the FC come from this matrix. With the new Lattice theory the solutions of problems 1-3 are straightforward, and all the possible symmetric FC can be defined with a given number of satellites.

\section{Constellation Design Considerations}

The 2D Lattice theory has then evolved into a 3D Lattice theory [14] allowing to design satellite constellations at any inclination using elliptical orbits and under the $J_{2}$ effect. The satellites phasing is obtained as solution of the $3 \times 3$ Hermite normal form

$$
\left[\begin{array}{ccc}
N_{o} & 0 & 0 \\
N_{c}^{3} & N_{\omega} & 0 \\
N_{c}^{1} & N_{c}^{2} & N_{s o}^{\prime}
\end{array}\right]\left\{\begin{array}{c}
\Omega_{i j k}-\Omega_{1} \\
\omega_{i j k}-\omega_{1} \\
M_{i j k}-M_{1}
\end{array}\right\}=2 \pi\left\{\begin{array}{l}
i \\
k \\
j
\end{array}\right\}
$$

However, designing an 3D Lattice Flower Constellation (3D-LFC) requires more than selecting the six integer parameters in Eq. (1). The semi-major axis $(a)$, eccentricity $(e)$, and inclination $(i)$ that are in common to all satellites must be selected. Additionally, the RAAN $\left(\Omega_{1}\right)$, argument of perigee $\left(\omega_{1}\right)$, and mean anomaly $\left(M_{1}\right)$ of the first reference satellite can also be selected arbitrarily without affecting the relative phasing within the constellation. 
Thus, an 3D-LFC requires six integer parameters and six continuous parameters. Essentially, the six continuous parameters define the orbit elements of the first satellite, and the six integer parameters phase all other satellites relative to that one. Each of the continuous parameters is subject to particular considerations as described in the following sections.

\section{A. Semi-major axis and eccentricity}

The orbit semi-major axis and eccentricity are common among all satellites in the constellation, and are typically bounded by some minimum and maximum altitudes. Typically these bounds are a result of sensor or antennae limitations. Requiring hardware that can operate at varying altitudes is a significant limitation on the use of elliptic orbits.

The semi-major axis can also be chosen to provide repeating ground-tracks as in the Walker or in the 2D-LFC theories. Satellites with the same argument of perigee can also be placed on the same repeating ground-track through judicious selection of the parameters $\left(N_{p}, N_{d}\right)$.

\section{B. Inclination}

The inclination of the orbits has significant impact on the coverage provided by a 3D-LFC. Even in circular orbit constellations, certain inclinations result in satellites colliding, whereas others permit near perfect phasing as a satellite from one plane passes directly between two satellites from another plane.

Considering two satellites in circular orbits with the same altitude, the closest approach between the two satellites, $\rho_{\min }$, can be analytically computed from the equations [26]

$$
\left\{\begin{aligned}
\Delta F & =\Delta M-2 \arctan [-\tan (\Delta \Omega / 2) \cos i] \\
\cos \beta & =\cos ^{2} i+\sin ^{2} i \cos \Delta \Omega \\
\rho_{\text {min }} & =2\left|\sqrt{\frac{1+\cos \beta}{2}} \sin \left(\frac{\Delta F}{2}\right)\right|
\end{aligned}\right.
$$

where $\Delta M$ and $\Delta \Omega$ are the difference in orbit elements of the two satellites and $i$ is the inclination angle common to both. Note that $\rho_{\min }$ must be scaled by the orbit radius to find the physical approach distance. The minimum distance encountered within a constellation of circular orbits can be computed by calculating this approach distance for all pairs of satellites. Perfect juggling requires that no two satellites are ever closer than half the distance between two consecutive satellites in the same orbit. We can scale the minimum approach distance such that zero corresponds to collision and one corresponds to perfect juggling. Using this scaling, the results for the 27/3/1 Walker constellation are plotted in Fig. 2 as a function of inclination angle. Note the peak near an inclination of $56^{\circ}$, the chosen inclination for the Galileo GNSS system [27]. This clearly indicates that even though inclination is technically a continuous parameter, there exist discrete values of inclination that maintain high levels of uniformity in the distribution of satellites. Equation (2) only applies to circular orbits, but similar derivations can be made for elliptic orbits with same value of perigee argument

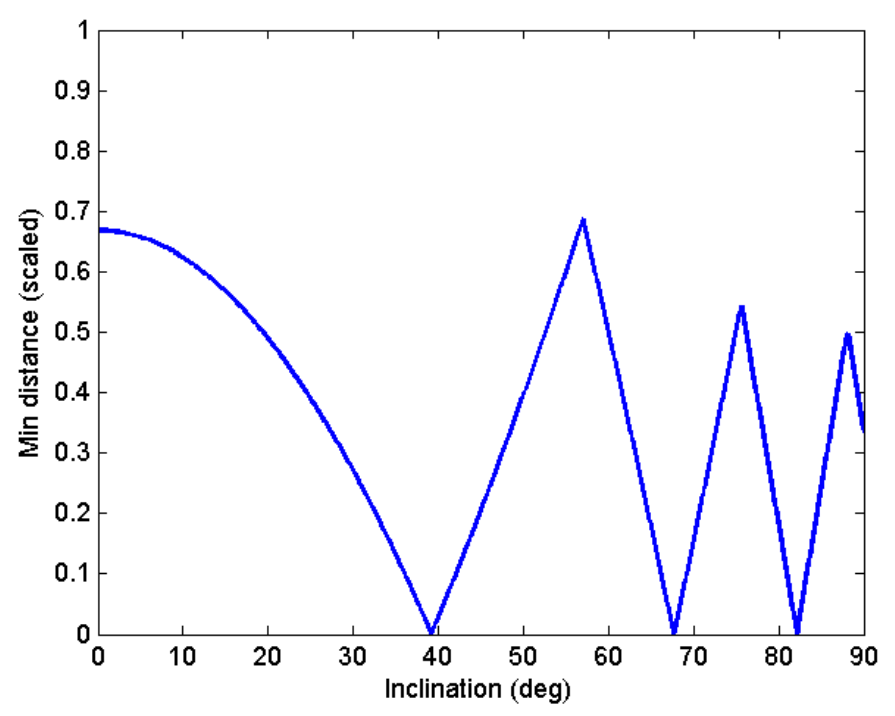

Fig. 2. Minimum encounter distance in the 27/3/1 Walker constellation as a function of inclination.

\section{Selecting $\Omega_{1}, \omega_{1}$, and $M_{1}$}

The values of $\left(\Omega_{1}, \omega_{1}, M_{1}\right)$ provide the three angular elements of the reference satellite $(i, j, k)=(0,0,0)$. Though each of them could be drawn from $[0,2 \pi)$, each can be further bounded by constellation considerations once the 6 integer parameters have been chosen.

Ref. ? has proven thal 3D-LFC can be described using values of $\left(\Omega_{1}, \omega_{1}, M_{1}\right)$ in the ranges

$$
\left\{\begin{aligned}
\Omega_{1} & \in\left[0, \frac{2 \pi}{N_{o}}\right) \\
\omega_{1} & \in\left[0, \frac{2 \pi}{N_{o} N_{\omega}} \operatorname{gcd}\left(N_{o}, N_{c}^{3}\right)\right) \\
M_{1} & \in\left[0, \frac{2 \pi}{N_{s}} \operatorname{gcd}\left(N_{o} N_{\omega}, N_{c}^{2} N_{o}, N_{\omega} N_{c}^{1}-N_{c}^{2} N_{c}^{3}\right)\right)
\end{aligned}\right.
$$

All 3D-LFC can be described by values within these ranges due to their uniform, symmetric nature. For general 3DLFC with a global coverage mission, the design parameters $\left(\Omega_{1}, \omega_{1}, M_{1}\right)$ can simply be taken as zero. When zonal or regional coverage is required, these variables significantly effect coverage. Clearly, $\omega_{1}$ is significant for critically inclined orbits, even when global coverage is considered, but has no meaning when dealing with circular orbits.

If one is considering global coverage, these ranges may not only be used for design purposes, they can also be used as limits on orbit propagations, thereby substantially reducing computation time. This is especially important given the rotation of the apsidal lines requires significantly more propagation than required for circular orbits. The optimal bounds can reduce the computation time by a factor of $N_{o}$ for $\omega$ and a factor of $N_{o} N_{\omega}$ for $M$ for a total possible reduction factor of $N_{o}^{2} N_{\omega}$. In the global navigation example of the next section, with 27 satellites in 3 orbital planes, the optimal 
bounds of Eq. (3) reduce propagation time by a factor of $\approx 7.5$ over these naive bounds when averaged over all 117 3D-LFC tested. Some of those 117 3D-LFC see a reduction in propagation time of a factor of $81\left(N_{o}=3, N_{\omega}=9\right)$. The 150 3D-LFC with 25 satellites and 5 orbital planes improve propagation times by a factor of $\approx 13.5$ on average, with a few improving by a factor of $125\left(N_{o}=5, N_{\omega}=5\right)$ !

To complete the picture with respect to other design methods, Walker's phasing parameter in Ref. [3] is equivalent to our $M_{1}$. Dufour includes an $\omega_{1}$ in his elliptical Walker constellations that is a multiple of another integer parameter he introduces, but the range of $\omega_{1}$ is limited to $[-\pi / 2, \pi / 2]$ rather than the full allowable range of $[-\pi, \pi]$ [24], [25]. The continuous parameter used here clearly includes the discrete values of Ref. [24], [25].

\section{Global Navigation Satellite System}

To examine the effectiveness of the 3D-LFC framework for designing a global coverage constellation, we first use the example of global navigation. Flower Constellations were first studied for use in GNSS by Park [19], who found improvements over the Galileo GNSS constellation by using a combination of two Harmonic Flower Constellations found by trial and error. Tonetti [22] ran a Genetic Algorithm (GA) to improve upon Park's results. Both of these Flower Constellations were designed for 30 satellites and utilized large numbers of orbital planes (15 and 30 respectively), which is unattractive from a launch and operational standpoint. Alternatively, Bruccoleri [21] found a Harmonic Flower Constellation with 24 satellites that showed improved performance over the GPS constellation. All three studies considered only circular orbits rather than be restricted to a critically inclined Flower Constellation with elliptic orbits. In this paper, in order to validate the proposed design methodology, we consider both 27 and 25 satellite 3D-LFC. We have not considered the combination of two or more 3D-LFC into the same constellation, as was done in Ref. [19], but this may yield additional improved results.

\section{A. Cost Function}

As a cost function to drive these design studies, we consider the Geometric Dilution Of Precision (GDOP), a measure of the accuracy of a GNSS solution. The lower the value of GDOP, the more accurate is the GNSS solution. GDOP is dependent entirely on the geometry of the satellites within view of a specific ground site and relies on the visibility matrix, given by

$$
A^{\mathrm{T}}=\left[\begin{array}{cccc}
\hat{\mathbf{r}}_{1} & \hat{\mathbf{r}}_{2} & \cdots & \hat{\mathbf{r}}_{n} \\
1 & 1 & \cdots & 1
\end{array}\right]
$$

where $\hat{\mathbf{r}}_{i}$ is the unit vector from ground site to the $i$-th satellite and $n$ is the number of visible satellites. We defined a minimum elevation angle of $10^{\circ}$ to determine satellite visibility in this simulation. We define the matrix $H=A^{\mathrm{T}} A$. GDOP can then be calculated

$$
\text { GDOP }=\sqrt{\operatorname{tr}\left(H^{-1}\right)} .
$$

This compact equation is simple, but requires a matrix inverse for every point (in time and space) that needs to be evaluated, so here we derive a new equation with faster computation. Since the trace of a matrix is the sum of its eigenvalues, and the eigenvalues of a matrix inverse are the inverses of the original matrix eigenvalues, we can rewrite the computation of GDOP as

$$
\mathrm{GDOP}=\sqrt{\sum_{i} \frac{1}{\lambda_{i}}}
$$

where $\lambda_{i}$ are the eigenvalues of $H$. Note that $\sum_{i} \lambda_{i}=2 n$. This alternate form of GDOP calculation reduced computation time in MATLAB by more than a factor of two.

To evaluate the accuracy of a given GNSS constellation, 1,000 points were distributed uniformly around a spherical Earth using an iterative electrostatic repulsion method (also known as the Thomson problem). The constellation was propagated using an initial argument of perigee of zero with $5^{\circ}$ steps in mean anomaly, and GDOP was calculated for all ground sites at each of those times. The initial argument of perigee was then rotated in $5^{\circ}$ steps with mean anomaly propagation performed at each step. This is a useful approximation of the behavior of the constellation due to the low rate of rotation of argument of perigee as compared to mean anomaly. The values of GDOP from all of these evaluations were then averaged, and we sought to minimize this mean GDOP value.

\section{B. Design Study: 27 Satellites}

In this paper, we compare performance to the Galileo constellation, designed as a 27/3/1 Walker constellation at $56^{\circ}$ inclination and semi-major axis of $29,600 \mathrm{~km}$ [27], [28]. Initial design studies based on a variety of performance and operational considerations led to this particular selection of the number of satellites and number of orbital planes, so those were held constant in this design paper. Once those numbers are fixed, the Walker constellation framework allows for just two design variables: the phasing parameter $F$ and the inclination angle. The phasing parameter is restricted to just 3 possible values. In contrast, the new 3D-LFC framework allows for 117 unique combinations of the parameters $\left\{N_{\omega}\right.$, $\left.N_{s o}^{\prime}, N_{c}^{1}, N_{c}^{2}, N_{c}^{3}\right\}$ and permits eccentricity to vary in addition to the inclination angle. Additionally, elliptic orbits are cheaper to launch into than circular orbits of the same semi-major axis, so holding launch cost constant allows 3D-LFC with higher altitudes. Thus, the search space is significantly expanded, yet still contains the original Galileo constellation design.

Preliminary analysis to reduce the design space consisted of evaluating all 117 3D-LFC over four values of eccentricity and eleven values of inclination:

$e \in[0.1,0.2,0.3,0.4], \quad$ and $\quad i \in\left[45^{\circ}, 47^{\circ}, \cdots, 65^{\circ}\right]$.

Circular orbits were not considered because they all collapse to $\mathrm{C}$-LFC/Walker constellations. The inclination range was chosen to place the Galileo optimal inclination of $56^{\circ}$ in the middle. The semi-major axis was held fixed at $29,655 \mathrm{~km}$, 
corresponding to a repetition time of 17 orbits in 10 days. A satellite was considered in view if it was at least $10^{\circ}$ above the horizon (grazing angle).

The constellations were evaluated for both mean GDOP and maximum GDOP encountered throughout the propagation. As a first cut, only solutions with a maximum GDOP below 6 were accepted (corresponding to the original requirements for the GPS constellation [29], [30]). There were 9 3D-LFC out of the original 117 that satisfied this requirement at a variety of inclinations and eccentricities, all of the form

$$
\left[\begin{array}{ccc}
N_{o} & 0 & 0 \\
N_{c}^{3} & N_{\omega} & 0 \\
N_{c}^{1} & N_{c}^{2} & N_{s o}^{\prime}
\end{array}\right]=\left[\begin{array}{ccc}
3 & 0 & 0 \\
N_{c}^{3} & 9 & 0 \\
N_{c}^{1} & 0 & 1
\end{array}\right]
$$

All of the minima for mean GDOP occurred in the inclination range $i \in\left[53^{\circ}, 59^{\circ}\right]$ over the full range of eccentricity.

This initial analysis was completed at a fixed altitude, but one advantage of elliptical orbits is their ability to launch into larger orbits for the same launch cost. The GIOVE-A and GIOVE-B satellites, launched as test vehicles for Galileo, launched into $190 \mathrm{~km}$ altitude circular parking orbits at an inclination of $51.8^{\circ}$ [31]. They were then boosted into their final orbit using a simple two-burn maneuver. Using the limiting case of a $60^{\circ}$ final inclination, a minimum eccentricity required to launch into an orbit of a given semi-major axis with the same two-burn maneuver cost as Galileo can be calculated.

Following the design guidelines laid out by the Galileo constellation design engineers, we seek a constellation with a repeating ground-track with repetition times between 5 and 10 days. Shorter repetition times lead to the build up of perturbations as the satellites pass over the same gravitational disturbances repeatedly, whereas longer repetition times pose operational challenges. Given these limitations and the desire to keep the apogee below GEO, we selected nine values of semi-major axis. Table I shows the different values of semimajor axis, minimum eccentricity (for the same launch cost), and maximum eccentricity (for apogee below GEO). Only values of semi-major axis larger than the planned Galileo system were considered because Ref. [28] shows that performance improves as altitude increases (though with diminishing returns, and they considered only circular orbits).

TABLE I

VALUES OF SEMI-MAJOR AXIS USED FOR GNSS OPTIMIZATION

\begin{tabular}{ccccc}
\hline \hline$N_{p}$ & $N_{d}$ & $a(\mathrm{~km})$ & $e_{\min }$ & $e_{\max }$ \\
\hline 17 & 10 & 29,655 & 0.045 & 0.424 \\
13 & 8 & 30,561 & 0.078 & 0.382 \\
8 & 5 & 30,878 & 0.089 & 0.368 \\
11 & 7 & 31,252 & 0.101 & 0.351 \\
14 & 9 & 31,464 & 0.107 & 0.342 \\
13 & 9 & 33,057 & 0.151 & 0.277 \\
10 & 7 & 33,302 & 0.157 & 0.268 \\
7 & 5 & 33,753 & 0.168 & 0.251 \\
11 & 8 & 34,161 & 0.177 & 0.236 \\
\hline \hline
\end{tabular}

For the second stage of the design study, another brute force grid search was completed with inclination selected from $i \in$ $\left[52^{\circ}, 53^{\circ}, \cdots, 60^{\circ}\right]$, semi-major axis and $e=e_{\min }$ selected from Table I, and the 3D-LFC parameters selected from the 9 3D-LFC down-selected in the first stage.

After selecting the optimal inclination angle for each 3DLFC at each altitude, one 3D-LFC outperformed all others at all altitudes:

$$
\left[\begin{array}{ccc}
N_{o} & 0 & 0 \\
N_{c}^{3} & N_{\omega} & 0 \\
N_{c}^{1} & N_{c}^{2} & N_{s o}^{\prime}
\end{array}\right]=\left[\begin{array}{ccc}
3 & 0 & 0 \\
2 & 9 & 0 \\
0 & 0 & 1
\end{array}\right]
$$

As expected, the best performance occurred at the maximum altitude with $a=34,161 \mathrm{~km}$ and $e=0.177$. The optimal inclination was the same as that of Galileo: $56^{\circ}$. The mean GDOP of Galileo was calculated to be 2.32 , whereas the mean GDOP of this 3D-LFC designed constellation is 2.24 - an improvement of $3.5 \%$. Given an inclination of only $56^{\circ}$ (as opposed to $60^{\circ}$ ), the minimum eccentricity to achieve the same launch cost to this much larger orbit is 0.15 . The mean GDOP varies only slightly (by 0.005 ) over the allowable eccentricity range, so eccentricity can be chosen based on other considerations. For instance, small eccentricity is attractive from an operational perspective, whereas larger eccentricity increases the allowable on-orbit satellite dry mass.

This 3D-LFC exhibits an interesting property: the satellites share the same geometry of the Galileo constellation at all times, they simply vary in altitude over time. The geometry is not an exact match, as the rotation of the argument of perigee perturbs it somewhat, but the two constellations bear great resemblance to one another. This "breathing" behavior, where the 3D-LFC mimics a C-LFC but with varying altitude, will occur for any 3D-LFC of the form

$$
\left[\begin{array}{ccc}
N_{o} & 0 & 0 \\
N_{c}^{3} & N_{\omega} & 0 \\
N_{c}^{1} & N_{c}^{2} & N_{s o}^{\prime}
\end{array}\right]=\left[\begin{array}{ccc}
N_{o} & 0 & 0 \\
N_{c} & N_{s o} & 0 \\
0 & 0 & 1
\end{array}\right]
$$

where $N_{o}, N_{s o}$, and $N_{c}$ are the parameters of the associated C-LFC.

The results of this study indicate that the Galileo constellation, at a semi-major axis of almost $30,000 \mathrm{~km}$ and 27 satellites, is very nearly optimal. The original designers chose a design point near the knee of the curve where increasing number of satellites or altitude met with diminishing returns [27], which is why the 3D-LFC design could only slightly improve upon the original Galileo design.

\section{Design Study: 25 Satellites}

The ultimate goal of a constellation designer is a constellation that maximizes performance while minimizing total system cost. Toward that end, reducing the number of satellites in a constellation, thereby eliminating its hardware and launch vehicle costs is one of the most effective means of reducing costs. We consider here the problem of designing an 3D-LFC with 25 satellites, divided into 5 orbital planes, to see what performance can be achieved while reducing the number of satellites by two. 
The design approach is the same as in the previous section. For the 25 satellite, 5 plane case, there exist 150 unique 3DLFC, and these were all studied over a range of eccentricities and inclinations at the original Galileo altitude. The maximum GDOPs encountered by the 25 satellite constellations were significantly higher than the original 27 satellite study, so the initial results were pared down by requiring the mean GDOP to be less than 3 and the maximum GDOP to be less than 16. This left 8 different 3D-LFC which were effective at a variety of eccentricities and inclinations. Table II shows the configuration parameters for these 8 3D-LFC, all of which had $N_{\omega}=5$.

TABLE II

ELLIPTICAL FLOWER CONSTELLATION PARAMETERS FOR 25 SATELLITE GNSS

\begin{tabular}{lcc}
\hline \hline$N_{c}^{1}$ & $N_{c}^{2}$ & $N_{c}^{3}$ \\
\hline 0 & 2 & 3 \\
3 & 2 & 2 \\
4 & 2 & 2 \\
3 & 3 & 4 \\
4 & 3 & 3 \\
3 & 4 & 3 \\
4 & 4 & 2 \\
4 & 4 & 4 \\
\hline \hline
\end{tabular}

When the altitude was allowed to vary as in the previous section (with $e=e_{\min }$ ), the maximum altitude was again the most effective. Unlike the 27 satellite case, however, there was significant variation in GDOP as a function of eccentricity, so each of the $83 \mathrm{D}$-LFC were analyzed over a range of eccentricities at the maximum altitude.

The best 25 satellite constellation was found to be inclined at $55^{\circ}$ with an eccentricity of 0.207 and 3D-LFC parameters

$$
\left[\begin{array}{ccc}
N_{o} & 0 & 0 \\
N_{c}^{3} & N_{\omega} & 0 \\
N_{c}^{1} & N_{c}^{2} & N_{s o}^{\prime}
\end{array}\right]=\left[\begin{array}{ccc}
5 & 0 & 0 \\
4 & 5 & 0 \\
4 & 4 & 1
\end{array}\right]
$$

The mean GDOP experienced with this constellation was 2.49 , compared to the 2.24 of the 27 satellite 3D-LFC in the previous section. This $10 \%$ reduction in mean accuracy is significant, but may be warranted given the reduced costs of a 25 satellite constellation. Of course, if the spare satellite strategy employed is to place one spare satellite in every orbital plane, then both the 27 satellite, 3 plane constellation and this 25 satellite, 5 plane constellation require 30 total satellites on orbit. The major shortcoming of the 25 satellite constellation is its maximum GDOP of 9.11, compared to a maximum GDOP of 3.87 for the 27 satellite 3D-LFC. A value above 6 is considered unusable [29], so there are times at which users would be unable to get a fix using this system.

\section{Acknowledgments}

This project was supported financially by the Science and Technology Development Fund (STDF), Egypt, Grant No 666.

\section{REFERENCES}

[1] Walker, J."Some Circular Orbit Patterns Providing Continuous Whole Earth Coverage," British Interplanetary Journal, Vol. Soc. 24, 1971, pp. 369-384.

[2] Walker, J."Satellite Constellations," British Interplanetary Journal, Vol. Soc. 37,1984 , pp. 559-572

[3] Walker, J. "Continuous Whole-Earth Coverage by Circular Orbit Satellite Patterns," Tech. Rep. 77044, Royal Aircraft Establishment, March 1977.

[4] J. Draim, "A Common Period Four-Satellite Continuous Coverage Constellation," AIAA/AAS Astrodynamics Specialists Conference, Williamsburg, VA, August 1986.

[5] J. Draim, "A Six Satellite Continuous Global Double Coverage Constellation," AIAA/AAS Astrodynamics Specialists Conference, Kalispell, MN, August 1987.

[6] J. Draim, "Continuous Global N-Tuple Coverage with (2N+2) Satellites," Journal of Guidance, Control, and Dynamics, Vol. 6, Jan-Feb 1991, pp. $17-23$.

[7] Draim, J."Elliptical Orbit MEO Constellations: A Cost-Effective Approach for Multi-Satellite Systems," Space Technology, Vol. 16, No. 1, 1996

[8] Draim, J.E., Inciardi, R., Cefola, P., Proulx, R., and Carter, D."Demonstration of the COBRA Teardrop Concept Using Two Smallsats in 8-hr Elliptic Orbits," USU Conference on Small Satellites, SSC01-II-3, 2001

[9] J. Draim, "Satellite Constellations: The Breakwell Memorial Lecture," Proceedings of the 55th International Astronautical Congress, Vancouver, Canada, 2004.

[10] J.L. Junkins, J.L. and Engels, R.C."The Finite Element Approach in Gravity Modelling," Geodaetica (1979), Vol. 4, pp. 185-206

[11] Mortari, D., Wilkins, M.P., and Bruccoleri, C."The Flower Constellations," Journal of the Astronautical Sciences, Vol. 52, Nos. 1\&2, Jan.-June 2004, pp. 107-127.

[12] Avendaño, M.E., Davis, J.J., and Mortari, D. "The Lattice Theory of Flower Constellations," Proceedings of the 2010 Space Flight Mechanics Meeting Conference, San Diego, CA, February 2010.

[13] Avendaño, M.E., Davis, J.J., and Mortari, D. "The 2D Lattice Theory of Flower Constellations," Submitted to Celestrial Mechanics and Dynamic Astronomy.

[14] Davis, J.J., Avendaño, M.E., and Mortari, D. "The 3D Lattice Theory of Flower Constellations," Submitted to Celestrial Mechanics and Dynamic Astronomy.

[15] Henderson, T.A., Mortari, D., Junkins, J.L., and ño, M.E. "An Adaptive and Learning Approach to Sampling Optimization," 2009 Space Flight Mechanics Meeting Conference, Savannah, GA, Feb. 9-12, 2009.

[16] Spratling, B. and Mortari, D."The K-Vector ND and its Application to Building a Non-Dimensional Star-ID Catalog," 2009 Space Flight Mechanics Meeting, Savannah, GA, Feb. 9-12, 2009.

[17] D. Mortari"CASS: Responsive Space Using Flower Constellations and Periodic Close Encounters," AFRL contract. Dates: 06/01/08-05/30/09.

[18] M. Wilkins, C. Bruccoleri, and D. Mortari, "Constellation Design using Flower Constellations," Proceedings of the 2004 Space Flight Mechanics Meeting Conference, Maui, Hawaii, 2004.

[19] K. Park, M. Wilkins, and D. Mortari, "Uniformly Distributed Flower Constellation Design Study for Global Positioning System," Proceedings of the 2004 Space Flight Mechanics Meeting Conference, Maui, HI, 2004.

[20] G. Dutruel-Lecohier and M. B. Mora, "ORION-A Constellation Mission Analysis Tool," Mission design and implementation of satellite constellations, Proceedings of the International Workshop, Toulouse, France, 1998.

[21] C. Bruccoleri, Flower Constellation Optimization and Implementation. $\mathrm{PhD}$ thesis, Texas A\&M University, College Station, TX, December 2007.

[22] S. Tonetti, "Optimization of Flower Constellations: Applications in Global Navigation System and Space Interferometry," Proceedings of the 2009 AIAA Aerospace Sciences Meeting, Orlando, Florida, January 2009.

[23] D. Knuth, The Art of Computer Programming, Vol. 2. Reading, MA: Addison-Wesley, 1997.

[24] F. Dufour, "Coverage Optimization of Elliptical Satellite Constellations with an Extended Satellite Triplet Method," Proceedings of the 54th International Astronautical Congress, Bremen, Germany, October 2003.

[25] F. Dufour, "Optimal Continuous Coverage of the Northern Hemisphere with Elliptical Satellite Constellations," Proceedings of the 2004 Space Flight Mechanics Meeting Conference, Maui, Hawaii, February 2004. 
[26] L. Speckman, T. Lang, and W. Boyce, "An Analysis of the Line of Sight Vector Between Two Satellites in Common Altitude Circular Orbits," Proceedings of AIAA/AAS Astrodynamics Conference, Portland, OR, August 1990. AIAA-90-2988-CP.

[27] R. Piriz, B. Martin-Peiro, and M. Romay-Merino, "The Galileo Constellation Design: A Systematic Approach," Proceedings of the 18th International Technical Meeting of the Satellite Division of the Institute of Navigation, Long Beach, CA, September 2005.

[28] A. Mozo-Garcia, E. Herraiz-Monseco, A. Martin-Peiro, and M. RomayMerino, "Galileo Constellation Design," GPS Solutions, Vol. 4, April 2001, pp. 9-15.

[29] B. Parkinson and J. Spilker, Global Positioning System: Theory and Applications, Volume I. Washington, DC: American Institute of Aeronautics and Astronautics, 1996.

[30] B. Parkinson and J. Spilker, Global Positioning System: Theory and Applications, Volume II. Washington, DC: American Institute of Aeronautics and Astronautics, 1996.

[31] "Flight ST 21 Launch Kit (GIOVE-B)," http://www.starsem.com/news/kits.htm [retrieved 4 May 2010], April 2008.

[32] Avendaño, M.E. and Mortari, D. "New Insights on Flower Constellations Theory," IEEE Transactions on Aerospace and Electronic Systems, Vol. 48, No. 2, April 2012. 